\title{
Futility stopping in clinical trials
}

\author{
Pei He, Tze Leung Lai* and Olivia Y. LiaO
}

Early stopping due to futility, also referred to as a go/nogo decision, during interim analysis has become an important feature of clinical trial designs. Current methods for futility stopping in literature are mostly based on conditional power or predictive power in conjunction with the theory of stochastic curtailment or group sequential design. They have certain drawbacks that have been noted in literature. Herein we describe a new approach to futility stopping in clinical trial designs and the statistical theory underlying this approach. Simulation studies and theoretical analysis show the advantages of the approach in both parametric and nonparametric problems.

AMS 2000 SUBJECT CLASSIFICATIONS: Primary 62L05; secondary $62 \mathrm{~F} 03$.

KEYwORDS AND PHRASES: Conditional power, Efficient, Group sequential testing, Survival endpoint.

\section{INTRODUCTION}

Since the late 1970s, interim analyses of the accumulating data in a long-term clinical trial have become increasingly popular and are now standard in clinical trial designs to compare a new treatment with a control. While there have been many important advances in group sequential tests with prescribed type I error probability of falsely rejecting the null hypothesis that treatment is not better than control, theoretical developments in futility stopping (as opposed to early stopping for efficacy that may inflate the type I error) seem to have lagged behind. The seminal paper of Lan, Simon and Halperin [16] introduced the conditional power approach and provided a fundamental bound on the loss of power at a given alternative due to futility stopping by considering the conditional power at that alternative. The need to choose an alternative in the condtional power approach was subsequently circumvented by using the predictive power approach or by using maximum likelihood or other methods to estimate the actual parameter at interim analysis so that the estimate can be used to substitute for the alternative in the conditional power approach. Section 2 gives a review of the conditional power and predictive power approaches to futility stopping and certain issues with both approaches.

\footnotetext{
${ }^{*}$ Corresponding author.
}

Section 3 develops a statistical theory for futility stopping, which relates futility to evidence against the hypothesis that treatment is better than control by at least some prescribed amount. For testing the one-sided null hypothesis $H_{0}: u(\theta) \leq u_{0}$ in a multiparameter exponential family, this theory formulates futility stopping via rejection of the hypothesis $u(\theta) \geq u_{1}$, in which $u_{1}$ is an "implied alternative," that is, the alternative at which the reference test of $H_{0}$, with type I error $\alpha$ and taking no more than $M$ observations, has power $1-\hat{\alpha}$. Using this formulation, we describe in Section 3 a new methodology for futility stopping, first in the exponential family setting and then in nonparametric and semiparametric models. Further complications for timesequential trials with survival endpoints, which have been pointed out in $[15,18]$, are also addressed by an extension of the basic approach. Simulation studies are given in Section 4 and show the advantages of the proposed approach to futility stopping. Further discussion and concluding remarks are given in Section 5 .

\section{CONDITIONAL AND PREDICTIVE POWER APPROACHES}

The motivation underlying conditional and predictive power is to forecast the outcome of a given test, called a reference test, of a statistical hypothesis $H_{0}$ from the data $D_{t}$ up to the time $t$ when such a prediction is made. Since the outcome is binary (i.e. whether to reject $H_{0}$ or not), the forecast can be presented as the probability of rejecting $H_{0}$ at the end of the study given $D_{t}$. However, this probability has to be evaluated under some probability measure. In the context of hypothesis testing in a parametric family $\left\{P_{\theta}, \theta \in \Theta\right\},[16]$ proposed to consider the conditional power

$$
p_{t}(\theta)=P_{\theta}\left(\text { Reject } H_{0} \mid D_{t}\right) .
$$

Subsequently, [5] and [21] found it more appealing to put a prior distribution on $\theta$ and consider the posterior probability of rejecting $H_{0}$ at the end of the trial given $D_{t}$, and therefore advocated to consider the predictive power

$$
P_{t}=P\left(\text { Reject } H_{0} \mid D_{t}\right)=\int p_{t}(\theta) d \pi\left(\theta \mid D_{t}\right)
$$

where $\pi\left(\theta \mid D_{t}\right)$ is the posterior distribution of $\theta$. This idea had been proposed earlier by Herson [9].

For the problem of testing the one-sided hypothesis $H_{0}$ : $\theta \leq \theta_{0}$, the predictive power approach to futility stopping 
terminates the study if $P_{t} \leq \gamma$, for some threshold $0<\gamma<$ $\frac{1}{2}$, at time $t$ of interim analysis. Similarly the conditional power approach chooses an alternative $\theta_{1}>\theta_{0}$ and stops the study if $p_{t}\left(\theta_{1}\right) \leq \gamma$. It is shown in [16] that if the reference test, which may be sequential, has power $1-\beta$ at $\theta_{1}$, then adding such futility stopping feature to the test at all times of interim analysis still has power $\geq(1-\beta) /(1-\gamma)$; see also [12, p. 206-207].

While the conditional power approach to futility stopping requires specification of an alternative $\theta_{1}$, the predictive power approach requires specification of a prior distribution $\pi$. It is often difficult to come up with such a specification in practice. On the other hand, one can use $D_{t}$ to estimate the actual $\theta$ by maximum likelihood or other methods, as suggested by Lan and Wittes [17]. For normal observations $X_{i}$ with common unknown mean $\theta$ and known variance $\sigma^{2}$, using Lebesgue measure on the real line as the improper prior for $\theta$ yields the sample mean $\bar{X}_{t}$, as the posterior mean and also the MLE. In this case, for the fixed sample size test that reject $H_{0}: \theta=0$ if $\sqrt{n} \bar{X}_{n} \geq \sigma z_{\alpha}$, this predictive power is

$$
\Phi\left(\sqrt{\frac{t}{n-t}}\left(\frac{\sqrt{n}}{\sigma} \bar{X}_{t}-z_{\alpha}\right)\right)
$$

and the conditional power is

$$
p_{t}\left(\bar{X}_{t}\right)=\Phi\left(\sqrt{\frac{n}{n-t}}\left(\frac{\sqrt{n}}{\sigma} \bar{X}_{t}-z_{\alpha}\right)\right) ;
$$

see [12, p. 211]. Here and in the sequel, we use $\Phi$ to denote the standard normal distribution function and $z_{\alpha}=\Phi^{-1}(1-$ $\alpha)$.

Although using the conditional or predictive power to guide early stopping for futility is intuitively appealing, there is no statistical theory for such choice of the stopping criterion. In fact, using the MLE as the alternative already pre-supposes that the MLE falls outside the null hypothesis, and a widely used default option is to stop when the MLE belongs to $H_{0}$, which is consistent with (4) that falls below the type I error $\alpha$ in this case. However, this ignores the uncertainty in the estimate and can lose substantial power due to premature stopping, as shown in the simulation studies of $[2,3]$ on adaptive designs that use this kind of futility stopping. Pepe and Anderson [20] have proposed to adjust for this uncertainty by using $\bar{X}_{t}+\sigma / \sqrt{t}$ instead of $\bar{X}_{t}$ to substitute for $\theta_{1}$ in the conditional power approach.

Instead of estimating the alternative during interim analysis, one can focus on a particular alternative $\theta_{1}$ and consider the conditional power $p_{t}\left(\theta_{1}\right)$ or the predictive power with a prior distribution concentrated around $\theta_{1}$. Although [16] has shown that adding futility stopping to the reference test of $H_{0}: \theta \leq \theta_{0}$ if $p_{t}\left(\theta_{1}\right) \leq \gamma$ does not decrease the power of the reference test at $\theta_{1}$, by more than a factor of $\gamma /(1-\gamma)$, there is no statistical theory justifying why one should use a conditional instead of an unconditional test of $\theta \geq \theta_{1}$. Furthermore, as noted earlier, this approach leaves open the problem of how $\theta_{1}$ should be chosen for stopping a study due to futility.

\section{FUTILITY STOPPING THEORY}

In this section we develop a statistical theory of early stopping for futility. To fix the ideas, consider a confirmatory trial sponsored by a pharmaceutical company to demonstrate the efficacy of a new drug for its approval. The null hypothesis assumed by the regulatory agency is $H_{0}: u(\theta) \leq u_{0}$, where $\theta$ is a parameter vector and $u$ is a smooth function of $\theta$ so that $H_{0}$ represents that the treatment is not efficacious. For example, $\theta=\left(p_{1}, p_{2}\right)$ in the case of binary responses, where $p_{1}$ is the response probability of the new treatment and $p_{2}$ is that of the standard treatment, and $u(\theta)=p_{1}-p_{2}, u_{0}=0$. This formulation also includes nonparametric tests by allowing $\theta$ to be infinite-dimensional, e.g., $\theta=\left(F_{1}, F_{2}\right)$ with $F_{i}$ being the distribution function of the new $(i=1)$ or standard $(i=2)$ treatment. Here smoothness of $u$ means that it is compactly differentiable; see [6]. In Sections 3.1 and 3.2, we focus on the parametric case involving exponential families for which we can apply results from the theory of [14] on efficient group sequential tests. Section 3.3 provides extensions to nonparametric and semiparametric tests, and Section 3.4 addresses additional issues for time-sequential trials with survival endpoints.

\subsection{Alternative implied by the maximum sample size constraint}

Because of time and resource constraints, the sample size of a clinical trial cannot exceed some prescribed upper bound $M$. Lai and Shih [14] introduced the concept of an "implied alternative," which is the alternative implied by this constraint, for testing $H_{0}: \theta \leq \theta_{0}$ based on i.i.d. observations $X_{1}, X_{2}, \ldots$ from a one-paramter exponential family with density $f_{\theta}(x)=e^{\theta x-\psi(\theta)}$. In this setting, application of the Neyman-Pearson lemma yields the fixed sample size test that rejects $H_{0}$ if $S_{M} \geq c_{\alpha}$ as the uniformly most powerful (UMP) level- $\alpha$ test for every alternative $\theta>\theta_{0}$, where $S_{M}=\sum_{i=1}^{M} X_{i}$ and $c_{\alpha}$ is chosen such that $P_{\theta_{0}}\left(S_{M} \geq c_{\alpha}\right)=\alpha$. In particular, it has maximal power, among all level- $\alpha$ tests (including sequential ones) that take no more than $M$ observations, at the implied alternative $\theta(M)$ where the above level- $\alpha$ test has prescribed power $1-\tilde{\alpha}$. In fact, the sample size $M$ can be determined by $P_{\theta_{1}}\left(S_{M} \geq c_{\alpha}\right)$ when $\theta_{1}=\theta(M)$ is given.

The successful outcome of the trial, from the pharmaceutical company's viewpoint, is rejection of the one-sided hypothesis $H_{0}$ by using a test that maintains the specified type I error. Although stopping early for futility would not increase the type I error, it would decrease the power of the test. In paticular, adding futility stopping to the test that rejects $H_{0}$ if $S_{M} \geq c_{\alpha}$ would lose the UMP property. Since the UMP test has the desired power $1-\tilde{\alpha}$ at $\theta(M)$, we would 
like the power to be still around $1-\tilde{\alpha}$ at $\theta(M)$ when such futility stopping is introduced. Thus, in this one-parameter exponential family setting, one can study futility stopping via the problem of testing $H_{0}: \theta \leq \theta_{0}$ versus $H_{1}: \theta \geq \theta(M)$, with type I error $\alpha$ and type II error slightly more than $\tilde{\alpha}$ and taking no more than $M$ observations.

\subsection{Group sequential testing theory}

For the problem of testing $H_{0}: \theta \leq \theta_{0}$ versus $H_{1}: \theta \geq \theta_{1}$ in the one-parameter exponential family, Lai and Shih [14] start by extending Hoeffding's lower bound [10] for the expected sample size $E_{\theta}(T)$ with error probabilities $\alpha$ at $\theta_{0}$ and $\tilde{\alpha}$ at $\theta_{1}$ to the group sequential setting, in which stopping can only occur at times of interim analysis with sample sizes $n_{1}<\cdots<n_{k}=M$. They show that this lower bound can be attained asymptotically as the error probabilities approach 0 by a group sequential version of Lorden's 2-SPRT [19], which runs simultaneously the sequential probability ratio test (SPRT) of the simple null $\theta_{0}$ versus the simple alternative $\theta$ and the SPRT of $\theta_{1}$ versus $\theta$, and which stops as soon as one of the SPRTs rejects the corresponding null hypothesis. They then modify this test to allow sequential updating of $\theta$ by maximum likelihood during the course of the trial. The likelihood ratio of $\theta$ to $\theta_{j}$ is replaced by the generalized likelihood ratio (GLR) of $\hat{\theta}_{n_{i}}$ to $\theta_{j},(j=0,1)$ at the $i$ th interim analysis. In particular, the test stops early at the $i$ th interim analysis $(1 \leq i \leq k-1)$ and rejects $H_{1}: \theta \geq \theta_{1}$ if

$$
\hat{\theta}_{n_{i}}<\theta_{1} \text { and } n_{i} I\left(\hat{\theta}_{n_{i}}, \theta_{1}\right) \geq \tilde{b},
$$

where $\tilde{b}$ is chosen such that

$$
P_{\theta_{1}}\{(5) \text { holds for some } 1 \leq i \leq k-1\}=\epsilon \tilde{\alpha}
$$

for some $0<\epsilon<\frac{1}{2}$, in which $I(\theta, \lambda)$ is the Kullback-Leibler information number $(\theta-\lambda) \psi^{\prime}(\theta)-\{\psi(\theta)-\psi(\lambda)\}$ and therefore $n_{i} I\left(\hat{\theta}_{n_{i}}, \theta_{1}\right)$ is the logarithm of the GLR of $\hat{\theta}_{i}$ to $\theta_{1}$. Note that (5) can be regarded as testing $\theta_{1}$ against the MLE $\hat{\theta}_{n_{i}}<\theta_{1}$ and that rejection of $H_{1}$ is the same as acceptance of $H_{0}$, so the sequential GLR test of $H_{1}$ is used for futility stopping in testing $H_{0}$.

Early stopping for efficacy, with $H_{0}$ rejected, occurs if

$$
\hat{\theta}_{n_{i}}>\theta_{0} \text { and } n_{i} I\left(\hat{\theta}_{n_{i}}, \theta_{1}\right) \geq b .
$$

In case stopping does not occur in the first $k-1$ analyses, reject $H_{0}$ if $S_{n_{k}} \geq c$, where $b$ and $c$ are so chosen that the test has error probability $\alpha$ at $\theta_{0}$ of falsely rejecting $H_{0}$. Note that $S_{n_{k}} \geq c$ can be written in the form (7) with $i=k$ and $b$ replaced by $\tilde{c}$, and that (7) can be regarded as testing $\theta_{0}$ against the MLE $\hat{\theta}_{n_{i}}>\theta_{0}$. With $\theta_{1}=\theta(M)$, Theorem 3 of [14] shows that the test, which is called the modified Haybittle-Peto test, has asympototically minimal expected sample size $E_{\theta}(T)$ at the true parameter $\theta$ and has power $1-\tilde{\alpha}-\kappa_{\epsilon} \tilde{\alpha}+o(\tilde{\alpha})$ at $\theta_{1}$ as $\alpha+\tilde{\alpha} \rightarrow 0$, where $\kappa_{\epsilon}$ is a constant depending on $\epsilon$.

Section 3.4 of [14] has extended this group sequential testing theory to the multiparameter exponential family $f_{\theta}(x)=$ $e^{\theta^{T} x-\psi(\theta)}$ and to multi-arm settings. The null hypothesis has the more general form $H_{0}: u(\theta) \leq u_{0}$. The Kullback-leibler information number becomes $(\theta-\lambda)^{T} \nabla \psi(\theta)-\{\psi(\theta)-\psi(\lambda)\}$, where $\nabla$ is the gradient vector. Suppose $I(\theta, \lambda)$ is increasing in $|u(\lambda)-u(\theta)|$ for every fixed $\theta$. Then we can still define the alternative $u_{1}$ implied by the maximum sample size $M$ and the type II error probability $\tilde{\alpha}$ of the reference test. Let $u_{1}=u_{1}(M)$ be such tht the fixed sample size GLR test of $H_{0}$ with type I error probability $\alpha$ and sample size $M$ has power

$$
\inf _{\theta: u(\theta)=u_{1}} P_{\theta}\left(\text { GLR test rejects } H_{0}\right)=1-\tilde{\alpha} ;
$$

see [3, Section 2.1]. Therefore futility stopping can again be carried out as before, using the sequential GLR test of $H_{1}$ : $u(\theta) \geq u_{1}$. In multi-arm clinical trials, for which different numbers of patients are assigned to $I$ different treatments, the GLR statistic at the $j$ th interim analysis is $e^{\Lambda_{j}}$, where

$$
\begin{aligned}
\Lambda_{j}= & \sum_{i=1}^{I} n_{i j}\left\{\hat{\theta}_{i, n_{i j}}^{T} \bar{X}_{i, n_{i j}}-\psi\left(\hat{\theta}_{i, n_{i j}}\right)\right\} \\
& -\sup _{u\left(\theta_{1}, \ldots, \theta_{I}\right)=u_{0}} \sum_{i=1}^{I} n_{i j}\left\{\theta_{i}^{T} \bar{X}_{i, n_{i j}}-\psi\left(\theta_{i}\right)\right\},
\end{aligned}
$$

in which $n_{i j}$ is the total number of observations from the $i$ th population up to the time of the $j$ th interim analysis. Let $n_{j}=n_{1 j}+\cdots+n_{I j}$. The asymptotic theory for the modified Haybittle test is extended in Section 3.4 of [14] to the case that uses adaptive randomization such that $n_{i j}=p_{i} n_{j}+O_{p}\left(\sqrt{n_{j}}\right)$ under $u(\theta)=u_{0}$ or under $u(\theta)=u_{1}$, in which $p_{1}, \ldots, p_{I}$ are nonnegative constants that sum up to 1 and can differ for the cases $u_{0}$ and $u_{1}$. Zhu and $\mathrm{Hu}$ [23] have recently demonstrated the advantages of using adaptive randomization over traditional equal randomization in group sequential trials.

\subsection{Extensions to nonparametric and semiparametric tests}

In many confirmatory clinical trials, $M$ is large and the treatment effect is assumed to belong to the framework of "local alternatives" for sample size calculation that justifies the choice of $M$. These local alternatives lead to "locally asymptotically normal" (LAN) families for the sampling distributions of the parametric, or nonparametric, or semiparametric test statistics used; see [1]. Because of its proximity to $H_{0}$, the MLE of a local alternative has substaintial probability of falling in $H_{0}$, and therefore one can lose considerable power by replacing a local alternative by its MLE in the conditional power $p_{t}(\theta)$, as pointed out in Section 2. 
We first consider nonparametric group sequential tests of $H_{0}: u(F, G) \leq 0$, where $F$ is the distribution function of the outcome of a new treatment and $G$ is that of the standard treatment (or placebo), and $u(F, F)=0$. Let $n_{i}^{\prime}$ be the sample size of the new treatment and $n_{i}^{\prime \prime}$ be that of the standard treatment at the $i$ th interm analysis so that $n_{i}=n_{i}^{\prime}+n_{i}^{\prime \prime}$, and let $X_{1}, \ldots, X_{n_{i}^{\prime}}$ and $Y_{1}, \ldots, Y_{n_{i}^{\prime \prime}}$ be the corresponding outcomes. Let $\hat{F}_{n_{i}^{\prime}}$ be the empirical distribution function of $X_{1}, \ldots, X_{n_{i}^{\prime}}$, and $\hat{G}_{n_{i}^{\prime \prime}}$ be that of $Y_{1}, \ldots, Y_{n_{i}^{\prime \prime}}$. As shown in [13], commonly used two-sample nonparametric test statistics can be written in the form of a generalized Chernoff-Savage statistic

$$
T_{i}=\int_{-\infty}^{\infty} J_{i}\left(\hat{F}_{n_{i}^{\prime}}(x), \hat{G}_{n_{i}^{\prime \prime}}(x)\right) d \hat{F}_{n_{i}^{\prime}}(x),
$$

where $J_{i}:\left\{0,1 / n_{i}^{\prime}, 2 / n_{i}^{\prime} \ldots 1\right\} \times\left\{0,1 / n_{i}^{\prime \prime}, 2 / n_{i}^{\prime \prime} \ldots 1\right\} \rightarrow \mathbb{R}$ satisfies

$$
\frac{1}{n_{i}^{\prime}} \sum_{l=1}^{n_{i}^{\prime}} \sup _{y \in\left\{1 / n_{i}^{\prime \prime}, \ldots, 1\right\}}\left|J_{i}\left(\frac{1}{n_{i}^{\prime}}, y\right)-J\left(\frac{1}{n}, y\right)\right| \rightarrow 0
$$

as $n_{i}^{\prime} \rightarrow \infty$, and $J:[0,1] \times[0,1] \rightarrow \mathbb{R}$ is twice continuously differentiable except possibly at $(0,0)$ and $(1,1)$ and satisfies certain regularity conditions near $(0,0)$ and $(1,1)$. In this case, the function $u(F, G)$ in $H_{0}: u(F, G) \leq 0$ is given by

$$
u(F, G)=\int_{-\infty}^{\infty} J(F(x), G(x)) d F(x) .
$$

Since subjects are randomly assigned to the new or standard treatment,

$$
n_{i}^{\prime} / n_{i} \stackrel{p}{\rightarrow} 1 / 2 \text {, i.e., } n_{i}^{\prime \prime}=n_{i}^{\prime}\left(1+o_{p}(1)\right) .
$$

Under (12), $T_{i}$ has the representation

$$
\begin{aligned}
T_{i}= & u(F, G)+\frac{1}{n_{i}^{\prime}} \sum_{l=1}^{n_{i}^{\prime}}\left(\psi\left(X_{i}\right)-E \psi\left(X_{i}\right)\right) \\
& +\frac{1}{n_{i}^{\prime \prime}} \sum_{l=1}^{n_{i}^{\prime \prime}}\left(\psi^{*}\left(Y_{i}\right)-E \psi^{*}\left(Y_{i}\right)\right)+R_{i}
\end{aligned}
$$

where $R_{i}=o_{p}\left(1 / \sqrt{n_{i}^{\prime}}\right)$ and

$$
\begin{aligned}
\psi(x) & =J(F(x), G(x))-\int_{0}^{x} \frac{\partial J}{\partial x}(F(t), G(t)) d F(t), \\
\psi^{*}(y) & =-\int_{0}^{y} \frac{\partial J}{\partial y}(F(t), G(t)) d F(t)
\end{aligned}
$$

see [4] and [13], which sharpens the proof in [4], to obtain a stronger result on $R_{i}$. For the present application, the convergence result $R_{i}=o_{p}\left(1 / \sqrt{n_{i}^{\prime}}\right)$ suffices. Therefore, under equal randomization to the two treatments, $n_{i}^{\prime} T_{i}$ behaves asymptotically like a normal random walk under $H_{0}$ and under local alternatives, and the problem of testing $H_{0}: u(F, G) \leq 0$ versus $H_{1}: u(F, G) \geq \delta$ can be approximated by that of group sequential testing of $H_{0}^{\prime}: \mu \leq 0$ versus $H_{1}^{\prime}: \mu \geq \delta$ based on i.i.d. normal random variables $Z_{1}, Z_{2}, \ldots$ with mean $\mu=u(F, G)$ and variance

$$
\sigma^{2}=\operatorname{Var}_{F=G}(\psi(X))+\operatorname{Var}_{F=G}\left(\psi^{*}(Y)\right),
$$

where $\psi$ and $\psi^{*}$ are given by (14). Note that $F=G$ is the boundary case of $H_{0}$ and that $F(X)$ and $G(Y)$ are Uniform $(0,1)$ random variables. Under local alternatives, the asymptotic variance of $T_{i}$ is the same as that under $F=G$, and therefore the variance formula (15) still holds for local alternatives. Further details and examples are given in Section 4.2.

The limiting normal random walk model and the more general Gaussian process model with independent increments have been derived by [11] and [12, Chapter 11] for efficient score statistics in parametric and Cox regression models. We have shown above that the limiting joint distribution holds generally for nonparametric test statistics, which may not be efficient score statistics, under the null hypothesis and local alternatives. Similarly, in the normal linear models considered by Jennison and Turnbull [11, Section 3], we can replace the normal models for the random errors by nonparametric models, leading to semiparametric regression models. When the regression parameters are estimated by least squares in this case, the score statistics or Wald statistics used for testing the regression parameters are not efficient, but the asymptotic joint normality of the test statistics at interim analysis still holds for the local alternatives. Therefore the theory for futility stopping developed in the preceding section for the exponential family (which includes the normal family) can be extended to the nonparametric or semiparametric setting. This is analogous to how group sequential tests developed for the prototypical normal case are extended to more general settings in [12].

\subsection{Futility stopping in time-sequential trials with survival outcomes}

Scharfstein, Tsiatis and Robins [? ] have extended the preceding results under the null hypothesis and local alternatives to efficient time-sequential score tests in semiparametric models. In particular, in the Cox regression model with regression parameter $\beta$, the efficient score statistic $S_{n}(t)$, which is the first derivative of the partial likelihood ratio statistic with respect to $\beta$, is locally asymptotically normal. Here $n$ is the sample size and $t$ is the time of an interim analysis. Specifically, $n^{-1 / 2} S_{n}(t)$ converges in distribution, as $n \rightarrow \infty$, to a Gaussian process with independent increments with variance $V(t)$ under the null hypothesis $\beta=0$ and local alternatives. The mean of the limiting Gaussian process is 0 under $\beta=0$ and is $\delta V(t)$ under local alternatives in the proportional hazards (or Cox) regression model. Therefore, the null variance of $S_{n}\left(t_{i}\right)$, which is 
approximately $n V\left(t_{i}\right)$ at the $i$ th interim analysis takes the place of the sample size $n_{i}$ in the preceding discussion.

The power calculations at the design stage of a timesequential trial with survival endpoint typically assume a working model of survival functions $\bar{F}=1-F$ and $\bar{G}=$ $1-G$, the accrual pattern and the censoring rates per year. The working model embeds the null case $\bar{F}=\bar{G}$ in a semiparametric family whose parameters are fully specified for the alternative hypothesis, under which the study duration and sample size of the two-sample semiparametric test are shown to have some prescribed power. Illustrative examples are given in Section 4.3. The two-sample test statistic $S_{n}(t)$ is usually chosen to be an efficient score statistic or its asymptotic equivalent in the working model. Unlike the nonparametric two-sample test of $H_{0}: u(F, G) \leq 0$ considered above, in which the asymptotic variance of $n_{i}^{\prime} T_{i}$ is proportional to the sample size $n_{i}^{\prime} \approx n_{i} / 2$ at the $i$ th interim analysis in view of (13), the asymptotic null variance $n V\left(t_{i}\right)$ of $S_{n}\left(t_{i}\right)$ depends not only on the survival distribution but also on the accrual rate and the censoring distribution up to the time $t_{i}$ of the $i$ th interim analysis. The observed patterns, however, may differ substantially from those assumed in the working model for the power calculations at the design stage. In addition, the working model under which the test statistic is semiparametrically efficient (e.g., the proportional hazards model when a logrank test is used) may not actually hold. In this case, as the sample size $n$ approaches $\infty$, the limiting distribution of $\sqrt{n} S_{n}(t)$ is still normal with mean 0 and variance $V(t)$ under $F=G$ and has independent increments, but under local alternatives, the mean $\mu(t)$ of the limiting normal distribution of $\sqrt{n} S_{n}(t)$ may not be linear in $V(t)$, and may level off or even decrease with increasing $V(t)$; see [7].

For the futility stopping decision at interim analysis, we can consider local alternatives, which suggest using the test $H_{0}: \mu\left(t_{i}\right) \leq 0$ for $1 \leq i \leq k$ versus $H_{\delta}: \mu\left(t_{i}\right) \geq \delta V\left(t_{i}\right)$ for some $i$, as discussed in the first paragraph of this section. We choose the same $\delta$ as that used in the design stage to determine the sample size and trial duration, since we do not want to have substantial power loss at or near the alternative assumed at the design stage. Even when the working model does not actually hold, for which $\mu(t) / V(t)$ may vary with $t$, using it to determine the implied alternative for futility stopping only makes it more conservative to stop for futility because $\mu(t)$ tends to level off or even decrease instead of increasing linearly with $V(t)$. It remains to consider how to update, at the $i$ th interim analysis, the estimated value of the "maximum information" $n V\left(t^{*}\right)$ (and also $n V\left(t_{j}\right)$ for $j>i$ if the reference test is time-sequential) after observing accrual, censoring and survival patterns that differ substantially from those assumed at the design stage. Our strategy is to replace $V(t)$ by the estimated $\hat{V}(t)$ for $t>t_{i}$ in the efficient score test of $H_{\delta}$ that involves these values, but not to estimate $\mu(t)$ for $t>t_{i}$ because we are in the setting of local alternatives with small $\delta$ (of the order $1 / \sqrt{n}$ ).
Bayesian modeling provides a natural updating scheme for estimating at time $t_{i}$ of interim analysis based on observations up to $t_{i}$, the null variance $V_{n}(t)$ of the score statistic $S_{n}(t)$ for $t>t_{i}$. Following [22], we use Dirichlet process priors for the distribution function $(F+G) / 2$ and for the censoring (i.e., patient withdrawal or loss in follow-up) distribution. Note that the null variance $V_{n}(t)$ is generated by the accrual rate, the censoring distribution, and the survival distributions $F$ and $G$ that are assumed to be equal. The parameter $\alpha$, which is a finite measure on $\mathbb{R}_{+}=(0, \infty)$, of the Dirichlet process prior for $1-H$, where $H=(\bar{F}+\bar{G}) / 2$, can be chosen to be some constant times the assumed parametric model, that is used for power calculation at the design stage, where the constant is $\alpha\left(\mathbb{R}_{+}\right)$that reflects the strength of this prior measure relative to the sample data. At the $i$ th interim analysis, let $n_{i}$ be the total number of subjects who have been accrued and let

$$
Z_{j}^{(i)}=\min \left(T_{j}, \xi_{j}, t_{i}-\tau_{j}\right), \quad \delta_{j}^{(i)}=I_{\left\{Z_{j}^{(i)}=T_{j}\right\}},
$$

$j=1, \ldots, n_{i}$, where $T_{j}$ is the actual survival time of the $j$ th patient, $\tau_{j}$ is the patient's entry time and $\xi_{j}$ is the censoring time. By re-arranging the observations, we can assume without loss of generality that $Z_{1}^{(i)}, \ldots, Z_{k}^{(i)}$ are the uncensored observation, and let $Z_{[k+1]}^{(i)}<\cdots<Z_{[m]}^{(i)}$ denote the distinct ordered censored observations. Let

$$
\begin{aligned}
& N_{i}(u)=\sum_{j=1}^{n_{i}} I_{\left\{Z_{j}^{(i)} \geq u\right\}}, \quad N_{i}^{+}(u)=\sum_{j=1}^{n_{i}} I_{\left\{Z_{j}^{(i)}>u\right\}}, \\
& \lambda_{i}(u)=\sum_{j=1}^{n_{i}} I_{\left\{Z_{j}^{(i)}=u, \delta_{j}=0\right\}}, \quad Z_{[k]}^{(i)}=0, \quad Z_{[m+1]}^{(i)}=\infty .
\end{aligned}
$$

As shown in [22], for $Z_{[l]}^{(i)} \leq u<Z_{[l+1]}^{(i)}$, the Bayes estimate of $H(u)$ at the $i$ th interim analysis is given by

$$
\begin{aligned}
\hat{H}_{i}(u)= & \frac{\alpha(u, \infty)+N_{i}^{+}(u)}{\alpha\left(\mathbb{R}_{+}\right)+n_{i}} \\
& \times \prod_{j=k+1}^{l}\left\{\frac{\alpha\left[Z_{[j]}^{(i)}, \infty\right)+N_{i}\left(Z_{[j]}^{(i)}\right)}{\alpha\left[Z_{[j]}^{(i)}, \infty\right)+N_{i}\left(Z_{[j]}^{(i)}\right)-\lambda_{i}\left(Z_{[j]}^{(i)}\right)}\right\} .
\end{aligned}
$$

Similarly, for updating the estimate $\hat{C}$ of the censoring distribution, we can interchange the roles of $T_{j}$ and $\xi_{j}$ above and replace $\alpha$ by $\alpha_{c}$ that is associated with the specification of the censoring distribution at the design stage. The accrual rates for the period prior to $t_{i}$ have been observed and those for the future years can use what is assumed at the design stage. Since $V_{n}(t)=V_{n}\left(t_{i}\right)+\left[V_{n}(t)-V_{n}\left(t_{i}\right)\right]$, we can estimate $V_{n}(t)$ by $V_{n}\left(t_{i}\right)+\mathrm{E}\left[V_{n}^{*}(t)-V_{n}^{*}\left(t_{i}\right) \mid \hat{H}, \hat{C}\right]$, in which the expectation $\mathrm{E}$ assumes the updated accrual rates and can be computed by Monte Carlo simulations to generate the observations $\left(Z_{j}^{*}, \delta_{j}^{*}\right)$ that are independent of the 
Table 1. Comparative study in normal case

\begin{tabular}{|c|c|c|c|c|c|c|c|c|c|c|c|}
\hline \multirow[t]{2}{*}{$\theta$} & \multirow{2}{*}{$\begin{array}{c}\text { FSS } \\
\text { Power }\end{array}$} & \multicolumn{2}{|c|}{ CP(MLE) } & \multicolumn{2}{|c|}{ CP(MLE+se) } & \multicolumn{2}{|c|}{$\mathrm{PP}$} & \multicolumn{2}{|c|}{$\mathrm{GLR}_{f}$} & \multicolumn{2}{|c|}{$\mathrm{GLR}_{e, f}$} \\
\hline & & Power & $\mathrm{E}(\#)$ & Power & $\mathrm{E}(\#)$ & Power & $\mathrm{E}(\#)$ & Power & $\mathrm{E}(\#)$ & Power & $\mathrm{E}(\#)$ \\
\hline 0 & 0.05 & 0.03 & 1.60 & 0.04 & 2.32 & 0.03 & 1.79 & 0.05 & 3.15 & 0.05 & 3.10 \\
\hline$\theta_{1}=0.13$ & 0.80 & 0.57 & 3.58 & 0.72 & 4.41 & 0.62 & 3.88 & 0.79 & 4.80 & 0.78 & 3.74 \\
\hline $1.2 \theta_{1}$ & 0.91 & 0.69 & 3.95 & 0.84 & 4.64 & 0.75 & 4.21 & 0.90 & 4.90 & 0.89 & 3.42 \\
\hline $2 \theta_{1}$ & 1.00 & 0.95 & 4.81 & 0.99 & 4.97 & 0.97 & 4.89 & 1.00 & 5.00 & 1.00 & 1.97 \\
\hline
\end{tabular}

Table 2. Comparative study in nonparametric case

\begin{tabular}{|c|c|c|c|c|c|c|c|c|c|c|c|}
\hline \multirow[t]{2}{*}{$\theta$} & \multirow{2}{*}{$\begin{array}{c}\text { FSS } \\
\text { Power }\end{array}$} & \multicolumn{2}{|c|}{$\mathrm{CP}(\mathrm{MLE})$} & \multicolumn{2}{|c|}{$\mathrm{CP}(\mathrm{MLE}+\mathrm{se})$} & \multicolumn{2}{|c|}{$\mathrm{PP}$} & \multicolumn{2}{|c|}{$\mathrm{GLR}_{f}$} & \multicolumn{2}{|c|}{$\mathrm{GLR}_{e, f}$} \\
\hline & & Power & $\mathrm{E}(\#)$ & Power & $\mathrm{E}(\#)$ & Power & $\mathrm{E}(\#)$ & Power & $\mathrm{E}(\#)$ & Power & $\mathrm{E}(\#)$ \\
\hline 0 & 0.05 & 0.03 & 1.59 & 0.04 & 2.31 & 0.03 & 1.66 & 0.05 & 3.16 & 0.05 & 3.11 \\
\hline$\theta_{1}=0.13$ & 0.77 & 0.53 & 3.49 & 0.68 & 4.32 & 0.55 & 3.58 & 0.75 & 4.77 & 0.74 & 3.83 \\
\hline $1.2 \theta_{1}$ & 0.88 & 0.66 & 3.86 & 0.82 & 4.59 & 0.68 & 3.94 & 0.87 & 4.88 & 0.86 & 3.56 \\
\hline $2 \theta_{1}$ & 1.00 & 0.92 & 4.70 & 0.98 & 4.95 & 0.93 & 4.72 & 0.99 & 4.99 & 1.00 & 2.20 \\
\hline
\end{tabular}

Table 3. Time-sequential example

\begin{tabular}{|c|c|c|c|c|c|c|}
\hline & \multirow[t]{2}{*}{ Hazard rate of $F$} & \multirow{2}{*}{$\begin{array}{c}\text { FSS } \\
\text { Power }\end{array}$} & \multicolumn{2}{|c|}{$\mathrm{GLR}_{f}$} & \multicolumn{2}{|c|}{$\mathrm{GLR}_{e, f}$} \\
\hline & & & Power & $\mathrm{E}(\#)$ & Power & $\mathrm{E}(\#)$ \\
\hline (1) & $\lambda_{0}=1 / 3(F=G)$ & 0.05 & 0.05 & 3.35 & 0.05 & 3.31 \\
\hline (2) & $\lambda_{0} / 1.4$ & 0.82 & 0.81 & 4.90 & 0.80 & 3.72 \\
\hline$(3)$ & $\lambda_{0} / 1.5$ & 0.92 & 0.91 & 4.94 & 0.91 & 3.37 \\
\hline (4) & $\lambda_{0} / 1.65$ & 0.98 & 0.98 & 4.98 & 0.98 & 2.85 \\
\hline (5) & $\lambda_{0} / 4$ for $0 \leq s \leq 1$ & & & & & \\
\hline & $\lambda_{0}$ for $s>1$ & 0.93 & 0.93 & 5.00 & 0.97 & 1.55 \\
\hline (6) & $\lambda_{0} / 5$ for $0 \leq s \leq 1$ & & & & & \\
\hline & $\lambda_{0}$ for $s>1$ & 0.96 & 0.96 & 5.00 & 0.99 & 1.35 \\
\hline (7) & $\lambda_{0} / 4.5$ for $s \leq 1$ or $s \geq 6$, & & & & & \\
\hline & $\lambda_{0} / 0.9$ for $1<s<6$ & 0.84 & 0.84 & 5.00 & 0.95 & 1.55 \\
\hline
\end{tabular}

$\left(Z_{j}^{(i)}, \delta_{j}^{(i)}\right)$ observed up to time $t_{i}$. Note that we can use the limiting independent increments property of $V_{n}(t) / n$ to simplify these computations and those for the stopping boundaries.

\section{SIMULATION STUDIES}

This section describes simulation studies of the futility stopping approach developed in Section 3. The results are given in Tables 1, 2 and 3; each result is based on 10,000 simulations. The reference test has a fixed sample size. We also consider in the last column of each table the case where the test can stop early for either efficacy or futility. Tables 1 and 2 compare the proposed methods in Sections 3.2 and 3.3 with the conditional power methods of [17] and [20]. These conditional power methods are denoted by CP(MLE) and $\mathrm{CP}(\mathrm{MLE}+\mathrm{se})$, respectively, and use the stopping criterion $\mathrm{CP}(\cdot) \leq \gamma=0.3$ suggested by [20]. For comparison with the predictive power (PP) method described in Section 2, we replace conditional power by the predictive power associated with the flat prior. Table 3 considers the time-sequential setting in Section 3.4, for which the reference test has fixed sample size and study duration.

\subsection{A comparative study for the prototypical normal mean case}

This simulation study considers the prototypical normal case in which the outcomes of the treatment arm and the placebo arm follow $N\left(\mu_{X}, 1\right)$ and $N\left(\mu_{Y}, 1\right)$, respectively. The problem is to test $H_{0}: \theta=\mu_{X}-\mu_{Y} \leq 0$, with type I error $\alpha=0.05$ and power $1-\tilde{\alpha}=0.8$ when $\mu_{Y}=1.5$. The trial involves $M=1,000$ subjects randomized to both arms with probability $1 / 2$ each, and interim analyses are planned with $n_{i}=200,400,600$, and 800 subjects, for $i=1,2,3,4$. The fixed sample size test has power 0.8 at the alternative 0.13 , which is the implied alternative $\theta(M)$ and will be denoted simply by $\theta_{1}$. We apply the group sequential GLR test described in the first paragraph of Section 3.2 with $\epsilon=1 / 3$ to perform futility stopping. This test will be denoted by $\mathrm{GLR}_{f}$, in which the subscript $f$ stands for early stopping for futility. Similarly, GLR ,,$f$ denotes the group sequential GLR test involving both efficacy and futility stopping. For $\mathrm{GLR}_{e, f}$ we use a non-binding futility boundary that does not consider the possibility of futility stopping in determining the efficacy boundary. Table 1 gives the power and the expected number of groups $E(\#)$ for $\mathrm{GLR}_{f}$ or $\mathrm{GLR}_{e, f}$. 


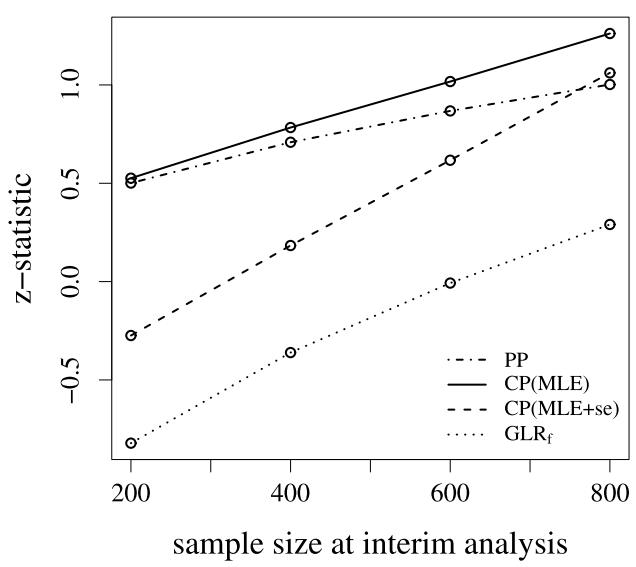

Figure 1. Futility stopping thresholds.

Since the group size is 200 , the expected sample size is $200 E(\#)$.

The first row $(\theta=0)$ of Table 1 shows that applying futility stopping reduces $E(\#)$ from 5 to $1.6-3.15$. The other rows show the performance of each test at the implied alternative $\theta_{1}$ and at the larger alternatives $1.2 \theta_{1}$ and $2 \theta_{1}$. Compared to the fixed sample size test, the group sequential GLR tests only lose $1 \%-2 \%$ power, while the conditional power and predictive power tests lose much more power, although they have smaller $E(\#)$. This fact is also illustrated in Fig. 1. The stopping criterion of the conditional or predictive power test and the group sequential $\mathrm{GLR}_{f}$ test can be transformed into thresholds for the standardized z-statistics

$$
\frac{\bar{X}_{n_{i} / 2}-\bar{Y}_{n_{i} / 2}}{\sqrt{4 / n_{i}}} \leq Z_{\mathcal{F}}\left(n_{i}\right),
$$

assuming approximately equal assignments of the $n_{i}$ subjects to the two treatments. In Fig. $1, Z_{\mathcal{F}}\left(n_{i}\right)$ is plotted against sample size $n_{i}$ for $\mathcal{F}=\mathrm{PP}, \mathrm{CP}(\mathrm{MLE})$, $\mathrm{CP}(\mathrm{MLE}+\mathrm{se}), \mathrm{GLR}_{f}$. It shows that $Z_{\mathcal{F}}\left(n_{i}\right)$ is markedly smaller for $\mathcal{F}=\mathrm{GLR}_{f}$ than the other methods and that $\mathrm{CP}(\mathrm{MLE}+\mathrm{se})$ has the second smallest value. Moreover, it is possbile to lower the threshold for $\mathrm{CP}(\mathrm{MLE}+\mathrm{se})$ by using a smaller value of the threshold $\gamma$ to trigger futility stopping when $\mathrm{CP}(\cdot)$ falls below the threshold. In fact, replacing $\gamma=0.3$ by $\gamma=0.05$ brings the performance of $\mathrm{CP}(\mathrm{MLE}+\mathrm{se})$ close to that of $\mathrm{GLR}_{f}$ in terms of power and $E(\#)$. Moreover, the steeper slope of $Z_{\mathrm{CP}(\mathrm{MLE}+\mathrm{se})}\left(n_{i}\right)$ reflects the adjustment for the uncertainty of the MLE, and such adjustment results in almost $20 \%$ more power than $\mathrm{CP}$ (MLE) in some cases.

\subsection{Futility stopping for two-sample Wilcoxon tests}

The second simulation study uses the fixed sample size Wilcoxon test of $H_{0}: F=G$ as the reference test. In view of (13), we can approximate the two-sample Wilcoxon statistic by a sum of normal random variables $Z_{1}, Z_{2}, \ldots$ with mean
$P(X<Y)$ and variance $1 / 12$ under $H_{0}$ and local alternatives. The simulation study uses the same design, sample size, and parameters as those described in Section 4.1, and assumes the distribution $F$ to be $\chi^{2}$ with 2 degrees of freedom, and $G$ to be a location shift of $F$, i.e., $G(x)=F(x-\theta)$. The implied alternative $\theta=0.182$ is the distance of the location shift, at which the fixed sample size test has power $80 \%$ using the aforementioned normal approximation. Table 2 shows results similar to those in Table 1, and in particular, that the conditional and predictive power tests tend to stop earlier for futility at the expense of substantial power loss when compared to the fixed sample size test.

\subsection{Futility stopping in time-sequential trials with survival outcomes}

The third simulation study is a continuation, with some modifications, of an example in [7]. The simulation study involves $n=450$ patients who arrive independently and uniformly over a 3-year interval and are randomized to the treatment and placebo arms. There are $k=5$ analyses at $1.5,2.5,3.5,4.5$ years and at 5.5 years when the trial is scheduled to end. Hence the expected study duration is the expected number of stages $E[\#]$ plus 0.5. Censoring due to loss of follow-up is assumed to be exponentially distributed with rate $1 / 6$. The failure-time distribution $G$ for the placebo arm is assumed to be exponential with hazard rate $\lambda_{0}=1 / 3$, so the median survival is of 3 years. Simulations are conducted under the null hypothesis $F=G$ (case 1) and under proportional hazards alternatives in which $F$ is exponential with hazard rate $\lambda=\lambda_{0} / 1.4, \lambda_{0} / 1.5, \lambda_{0} / 1.65$ (cases $2-4$ ). Besides proportional hazards alternatives, three other stochastically ordered alternatives are also considered and listed in Table 3 as cases $5-7$. In cases 5 and 6 , the hazard rate of $F$ is lower than that of $G$ in the first year. In case 7 , the hazard rate of $G$ only exceeds that of $F$ in years one to six.

Futility stopping via the sequential GLR method described in Section 3.4 saves almost half of the time in case 1, while losing less than 1 percent of the power compared to the fixed sample size and fixed duration test. For proportional hazards alternative, $\mathrm{GLR}_{e, f}$ has power similar to that of $\mathrm{GLR}_{f}$ but saves 1-2 stages (years), since the trial is allowed to stop early for efficacy. It even improves the power and reduces substantial study duration by $3.4-3.6$ years in cases $5-7$. This phenomenon has been explained by [7]; the expected value of $S_{n}(t)$ may actually decrease with increasing $t$, therefore allowing $\mathrm{GLR}_{e, f}$ to achieve both savings in time and increase in power over fixed-duration tests.

As pointed out in Section 3.4, our approach to futility stopping via $\mathrm{GLR}_{f}$ or $\mathrm{GLR}_{e, f}$ requires updating the estimate of $V\left(t^{*}\right)$, with $t^{*}=5.5$, at each interim analysis. We assume exponential densities $2,000 e^{-0.6 t}$ and $2,000 e^{-0.33 t}$ for the Dirichlet process priors of $(F+G) / 2$ and of the censoring distribution, respectively, for updating the Bayesian estimate $\hat{V}_{t_{i}}\left(t^{*}\right)$ at time $t_{i}$ of interim analysis, as described 


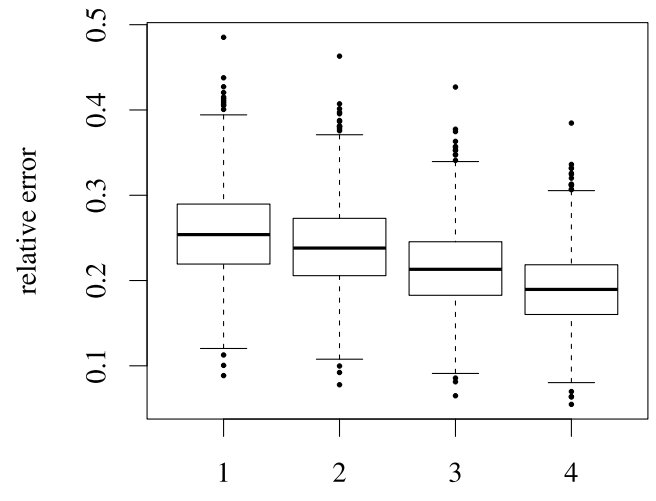

interim analysis index

Figure 2. Box-plots of relative errors of $\hat{V}_{t_{i}}\left(t^{*}\right)$.

in Section 3.4. To see how $\hat{V}_{t_{i}}\left(t^{*}\right)$ improves with increasing $i$ that accumulates more data, one can consider the relative error $\left|\hat{V}_{t_{i}}\left(t^{*}\right)-V\left(t^{*}\right)\right| / V\left(t^{*}\right)$. Figure 2 gives the box-plot of these relative errors over the 10,000 simulations for the $i$ th interim analysis, $i=1,2,3,4$, in case $1(F=G)$. It shows the decreasing trend of these relative errors with $i$. For the other cases, even though the relative errors become larger, with mean values ranging between 0.3 and 0.4 , their decreasing trend with $i$ are similar, showing that the estimates become more accurate with more data.

\section{DISCUSSION}

Unlike efficacy stopping, early stopping for futility during interim analyses does not inflate the type I error of the reference test. On the other hand, it may substantially decrease the power, or equivalently, substantially inflate the type II error if it is not carried out carefully. In particular, we have shown that the conditional power approach may have substantial power loss when it does not take into consideration the uncertainty of the estimated alternative. Whereas efficacy stopping is associated with rejection of $H_{0}: \theta \leq \theta_{0}$ at interim analysis, we can view futility stopping as rejection of the hypothesis $H_{\theta(M)}: \theta \geq \theta(M)$ associated with the implied alternative $\theta(M)$. This viewpoint enables us to use group sequential testing theory to develop a new approach to futility stopping. Simulation studies and asymptotic theory presented herein have shown its advantages over traditional conditional power and predictive power approaches, especially at the marginal alternatives.

Time sequential trials with survival outcomes have posed additional challenges to futility stopping. Lan and DeMets [15] have noted the difficulties due to the two time scales in these trials, namely calendar time and information time. The modified Haybittle-Peto approach we use in Section 3 circumvents the difficulties they cause for the commonly used error spending approach [12, Chapter 7]. As noted by Lin, Yao and Ying [18] for the logrank test, an additional challenge besides these two time scales is that the number of failure events observed during the course of the trial can substantially differ from that assumed at the design stage for determining the sample size and study duration of the trial, and it is useful to re-estimate this number, or more generally the maximum information $V\left(t^{*}\right)$ in the setting of Section 3.4, for futility decisions at interim analysis. Another useful contribution of the paper, therefore, is the new method proposed in Section 3.4 for updating the estimates of $V\left(t^{*}\right)$.

\section{ACKNOWLEDGEMENTS}

T. L. Lai's research was supported by the National Science Foundation grant DMS-085879 and the National Cancer Institute grant 1 P30 CA 124435-01. The research of Pei He and Olivia Liao was supported by the National Institutes of Health grant 4R37EB002784.

\section{Received 25 October 2011}

\section{REFERENCES}

[1] Andersen, P. K., Borgan, O., Gill, R. D., and Keiding, N. (1993). Statistical Models Based on Counting Process. Springer, New York. MR1198884

[2] Bartroff, J., and Lai, T. L. (2008a). Efficient adaptive designs with mid-course sample size adjustment in clinical trials. Stat. Med. 27, 1593-1611. MR2420330

[3] Bartroff, J., and Lai, T. L. (2008b). Generalized likelihood ratio statistics and uncertainty adjustments in efficient adaptive design of clinical trials. Sequential Anal. 27, 254-276. MR2446902

[4] Chernoff, H., and Savage, R. (1958). Asymptotic normality and efficiency of certain nonparametric test statistics. Ann. Math. Statist. 29, 972-994. MR0100322

[5] Choi, S. C., Smith, P. J., and Becker, D. P. (1985). Early decision in clinical trials when treatment differences are small. Contr. Clin. Trials. 6, 280-288.

[6] GILl, R. D. (1989). Non- and semi-parametric maximum likelihood estimators and the von Mises method. I. Scand. J. Statist. 16, 97-128. MR1028971

[7] GU, M., and LAI, T. L. (1998). Repeated significance testing with censored rank statistics in interim analysis of clinical trials. Statistica Sinica 8, 411-428. MR1624347

[8] Halperin, M., Lan, K. K. G., Ware, J. H., Johnson, N. J., and DeMets, D. L. (1982). An aid to data monitoring in long-term clinical trials. Contr. Clin. Trials. 3, 311-323.

[9] Herson, J. (1979). Predictive probability early termination plans for Phase II clinical trials. Biometrics 35, 775-783.

[10] Hoeffinin, W. (1960). Lower bounds for the expected sample size and the average risk of a sequential procedure. Ann. Math. Statist. 31, 352-368. MR0120750

[11] Jennison, C., and Turbull, B. W. (1997). Group-sequential analysis incorporating covariate information. J. Amer. Statist. Assoc. 92, 1330-1341. MR1615245

[12] Jennison, C., and Turbull, B. W. (2000). Group Sequential Methods 1. Chapman \& Hall, New York.

[13] LAI, T. L. (1975). Cherno-Savage statistics and sequential rank tests. Ann. Statist. 3, 825-845. MR0388615

[14] Lai, T. L., and Shin, M. (2004). Power, sample size and adaptation considerations in the design of group sequential clinical trials. Biometrika 3, 57-528. MR2090619

[15] Lan, K. K. G., and DeMets, D. L. (1989). Group sequential procedures: Calendar versus information time. Statist. Med. 8, 1191-1198. 
[16] Lan, K. K. G., Simon, R., and Halperin, M. (1982). Stochastically curtailed tests in long-term clinical trials theory. Commun. Statist. C 1, 207-219. MR0685474

[17] Lan, K. K. G., and Wittes, J. (1988). The B-value: A tool for monitoring data. Biometrics 44, 579-585.

[18] Lin, D. Y., YAO, Q., and Ying, Z. (1999). A general theory on stochastic curtailment for censored survival data. Joul. Amer. Statist. Assoc. 94, 510-521. MR1702321

[19] Lorden, G. (1976). 2-SPRTs and the modied Kiefer-Weiss problem of minimizing an expected sample size. Ann. Statist. 4, 281291. MR0405750

[20] Pepe, M. S., and Anderson, G. L. (1992). Two-stage experimental designs: Early stopping with a negative result. App. Statist. 41, 181-190.

[21] Spiegelhalter, D. J., Freedman, L. S., and Blackburn, P. R. (1986). Monitoring clinical trials: Conditional or predictive power? Contr. Clin. Trials. 7, 8-17.

[22] Susarla, V., and Ryzin, J. V. (1976). Nonparametric bayesian estimation of survival curves from incomplete observations. $J$ Amer. Statist. Assoc. 71, 897-902. MR0436445

[23] ZHu, H., and Hu, F. (2010). Sequential monitoring of responseadaptive randomized clinical trials. Ann. Statist. 38, 2218-2241. MR2676888
Pei He

390 Serra Mall Sequoia Hall

Stanford University, Stanford, CA 94305

USA

E-mail address: hepei@stanford.edu

Tze Leung Lai

390 Serra Mall Sequoia Hall

Stanford University, Stanford, CA 94305

USA

E-mail address: lait@stanford.edu

Olivia Y. Liao

390 Serra Mall Sequoia Hall

Stanford University, Stanford, CA 94305

USA

E-mail address: yuehwen@stanford.edu 